In addition to the localization related and generalized epilepsies and syndromes there are III. unclassified epilepsies, and IV. special syndromes, e.g. febrile convulsions. There are two appendices to the revised classification. 1. Symptamatic generalized epilepsies of specific etiologies including malformations and inborn errors of metabolism and Appendix 2 precipitated seizures, e.g. reflex epilepsies, startle epilepsy, and primary reading epilepsy. (Conmission on Classification and Terminology of the International League Against Epilepsy. Proposal for revised classification of epilepsies and epileptic syndromes. Epilepsia July/August 1989; 30:389-400).

COMIENT. The Commission recognizes that the revised classification is not totally satisfactory. Patients may move from one syndrome to another during the evolution of the epilepsy, e.g. a child with West syndrome may later satisfy the criteria for the Lennox-Gastaut syndrome. It is of interest that the Commission is reverting to the older traditional concept of an idiopathic "primary" epilepsy of presumed genetic etiology as separate from the cryptogenic epilepsies presumed to be symptamatic. Criticisms of the new classification not stressed by the Commission are as follows: 1) The grouping of seizure patterns proposed are at variance with therapeutic correlations, e.g. absence and tonic-clonic seizures grouped together as generalized epilepsies require different types of medication for their control, and 2) The oversimplification and emphasis of syndromes might lead to a decreased awareness of etiologies and misdiagnoses of underlying pathologies.

\title{
DRIUG-INDUCED SEIZURES
}

Recreational drug-induced seizures in 47 patients seen at the San Francisco General Hospital between 1975 and 1987 were reported by the Division of $\mathrm{Clinical}$ Pharmacy and Department of Neurology, University of California, San Francisco, CA. Over the twelve year study period 49 episodes of seizures followed the use of most of the popular street drugs and were seen after ingestion, snorting, smoking and injection. The average age of the 28 men and 19 wamen was 27 years (range 19-42 years). The majority of patients experienced a single generalized tonic-clonic seizure but seven had multiple seizures and two developed status epilepticus. The recreational drugs were cocaine (32), amphetamines (11), heroin (7), and phencyclidine (4). A combination was responsible in 11 . Seizures occurred independent of the route of administration and in both first-time and chronic abusers. Ten (21\%) had prior seizures, all closely associated with drug abuse. Apart from the patient with status epilepticus, none had permanent neurologic impairment at the time of discharge from hospital. (Alldredge BK et al. Seizures associated with recreational drug abuse. Neurology August 1989; 39:1037-1039).

COMNENT. Although the patients in this study were adults, the possibility of drug abuse should be considered in the evaluation of infants, children and adolescents with seizures. Heroin withdrawal has been associated with seizures in neonates (Herzlinger RA et al. J Pediatr $1977 ; \underline{91}: 638$ ) but not in older patients. It is fortunate 
that seizures due to recreational drug abuse appear to be selflimiting and not associated with neurologic sequelae. Anticonvulsant treatment is usually not required.

\section{CNS INFECTIONS}

LYME DISEASE

Six patients with central nervous system manifestations of Lyme disease are reported from the Department of Neurology, Georgetown University School of Medicine, Washington, DC. Behavioral changes, ataxia, and/or weakness in bulbar or peripheral muscles developed weeks to years after the initial infection. Four patients had lymphocytic pleocytosis in the CSF and two had MRI evidence of denyelination. All patients had elevated antibody titers to $B$ burgdorferi in serum and all were treated with high-dose intravenous penicillin. Four had recovered completely within one to three months. One patient had persistent brief episodes of vestibular neuronitis and optic neuritis 15 months after antibiotic therapy for myelitis. One patient in whom antibiotic therapy had been delayed for two years after the onset of CSF abnormalities failed to respond to repeated courses of IV penicillin and showed a progressive neurologic involvement with bilateral peripheral facial weakness, double vision, weakness of triceps, wrist and finger flexors and loss of sensation in hands and arms. (Pachner AR et al. Central nervous system manifestations of Lyme disease. Arch Neurol July 1989; 46:790-795).

COMNENT. A case of latent Lyme neuroborreliosis is reported in a $1 \overline{7 \text { year }}$ old boy from the University of Munich, Germany (Pfister H-W et al. Neurology August $1989 ; 39: 1118)$. Borrelia burgdorferi was isolated from the CSF, serum IgG antibody titers were elevated, but concurrent inflammatory signs of CSF as well as intrathecal antibody production were absent. Bilateral tinnitus was the only clinical symptom and this could not definitely be attributed to the Borrelial infection.

Lyme disease is the subject of Medical Progress (Steere AC. N Engl $\mathrm{J}$ Med August 31,$1989 ; 321: 386)$. Lyme disease commonly begins in sumer with a characteristic skin lesion, erythema migrans, accompanied by flu-like or meningitis-like symptams. Weeks or months later the patient may have neurologic or cardiac abnormalities, migratory musculoskeletal pain or arthritis. After the first several weeks of infection almost all patients have a positive antibody response to the spirochete and serologic determinations are currently considered the most practical laboratory aid in diagnosis. The author concludes that appropriate antibiotics are usually curative but longer courses of therapy are often needed later in the illness and some patients may not respond. The fetus may be at risk in mothers treated for the disease; a pregnant waman in Europe whose erythema migrans was treated with oral antibiotics gave birth to an infant who died of Lyme encephalitis. (Weber K et al. Pediatr Infect Dis J 1988; $\underline{7}: 286$ ). 